\title{
Science, Passion \& Compassion vs. Cancer: Tania Crombet MD PhD, Director of Clinical Research Molecular Immunology Center, Havana
}

\section{Conner Gorry MA}

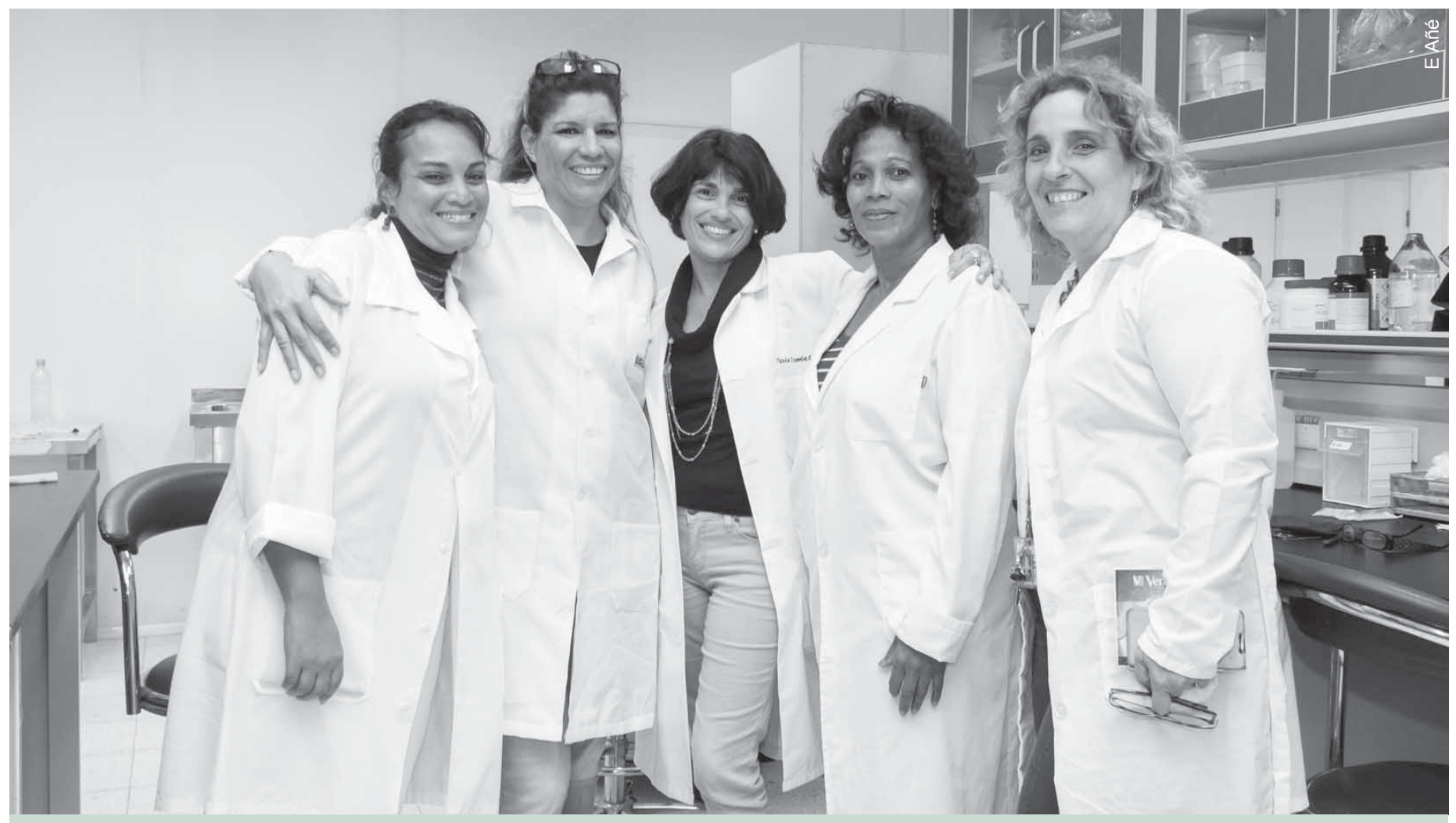

Dr Crombet (center) with her team at CIM

Soon after the Molecular Immunology Center (CIM) was established in 1994 (a founding institution of Havana's biotechnology and pharmaceutical campus known as the scientific pole), Dr Crombet completed her master's thesis there. She joined CIM's team in 1998 and in 2004 was designated Director of Clinical Research. She has participated in the research, development and clinical trials of some of Cuba's most innovative therapies and vaccines, including CIMAvax-EGF for non-small cell lung cancer patients. In 2015, this therapy completed Phase IV clinical trials in Cuba and is now used in primary health care services throughout the country's national health system. CIM and Roswell Park Cancer Institute (Buffalo,
New York) received US Department of Treasury approval in 2015 to test CIMAvax-EGF and other CIM products in the United States, opening the way for the Food and Drug Administration (FDA) to consider joint ground-breaking Phase I and II clinical trials in the USA. Recent regulatory changes introduced by President Barack Obama may make applying for such licenses a thing of the past-at least that is what researchers hope.

In any case, the work of Dr Crombet and the teams at CIM is making headway in cancer immunotherapy, within the broader goals of the institution's mandate...the subject of our interview.
MEDICC Review: Can you talk a little about CIM's history and its research objectives?

Tania Crombet: CIM was founded with the primary goal of developing safe, effective vaccines and therapies and, after successfully completing trials, registering them for use in the national health system. Our approach in all our scientific research is to forge solutions to our greatest health problems, so providing safe and effective treatments for the people who need them drives us and defines our priorities. Today there are nearly 1200 people working at CIM to find cures and develop therapies to help patients, not only in Cuba, but elsewhere in the world where CIM products are available. CIM follows strict Good Clinical Practice, Good Manufacturing Practice and Good Laboratory Practice standards. To assure we're in compliance, we undergo regular inspections. It seems we're constantly being inspected! As we should be. Our work also contributes to import substitution, an ongoing strategy for sustainable development of our health system. 


\section{Interview}

\section{MEDICC Review: So what are Cuba's biggest health problems and what role has CIM played in helping address them?}

Tania Crombet: For the last few years, cancer has alternated with cardiovascular disease as the number one cause of death in Cuba. Until now, CIM's product portfolio has been focused mainly on cancer: about $80 \%$ of our therapies and vaccines are for cancer-lung, breast, head and neck, prostate and more. However, we've recently started research on other chronic non-communicable diseases contributing to morbidity and mortality in Cuba; these efforts will intensify in 2017, altering CIM's research balance between cancer and other diseases.

For instance, neurological conditions, including dementia and Parkinsons, are becoming a bigger part of our portfolio and are important lines of research given the aging of the Cuban population. Likewise, we're conducting research on diabetes in adults, another chronic disease of concern here and elsewhere. So neurological and cardiovascular diseases - to treat spinal cerebellar ataxia and stroke for instance-are increasingly a more robust part of our research. This is a scientific challenge for several reasons, including participant selection for trials, since patients may be taking medications for other conditions that can interact with the candidate therapies being tested.

\section{MEDICC Review: What else is new at CIM?}

Tania Crombet: After 10 years of cross-sector collaboration among $\mathrm{CIM}$, the Informatics University ( $\mathrm{UCl}$ ) and Infomed [Cuba's national health telematics network, Eds.], our computer platform digitizing all clinical trial records was implemented throughout our clinical sites in June 2015. Paper records no longer exist at these sites, where use of the program is now compulsory. We've arrived at the 21st century! Seriously though, one of our strengths in Cuba is data collection and analysis and our clinical trial management software, Xavia-Clínicas, has not only improved the reliability and accuracy of that data, but has made our work more efficient and cost effective. We're already seeing the savings digital recordkeeping and transfer represent.

Before Xavia-Clínicas, we used a double-entry system, transcribing data from paper records to the main and backup databases. This information was checked and double checked. While rigorous, the system could introduce errors. Spelling mistakes for example, were inevitable, and you know doctors are famous for their legible handwriting! It was also painstakingly slow. So Xavia-Clínicas is much more reliable because it is designed to catch errors and has an internal structure that prevents users, among other things, from entering a number if it falls outside the established range. For instance, if a numeric value is entered for hemoglobin levels beyond the range contemplated in the study, the program won't permit it. Furthermore, Xavia-Clínicas stores all records with access levels determined according to interna- tional norms of privacy, confidentiality and ethics. These include signed informed-consent forms from clinical trial participants. The entire database is backed up daily on our server, again at week's end, and once a month on the Ministry of Public Health's (MINSAP) server.

About 600 users across the country were trained on Xavia-Clínicas by our team of scientific informatics specialists and $\mathrm{UCl}$ program designers. At first there was apprehension, fear even, about the new program-remember we're dealing here with health professionals, not IT specialists. What's more, we've collaborated in other countries where the clinical data program is so complicated and takes so long to learn, users get frustrated and end up rejecting the new technology.

So, with this in mind, Xavia-Clínicas was designed to be an extremely user-friendly platform (working on tablets, too), and doctors and staff became eager adopters after the first training sessions. Now they can't imagine going back to paper records. This slow, but smooth transition to digital records is a testament to the enthusiasm and energy of our IT team: they traveled the country from one end to the other, working 10-hour days with people who were afraid of the technology. And we're proud to say the program is showing results; it's one thing to design and install a program and say, 'we've done it. The program is up and running.' It's another to have it used by hundreds of specialists on a daily basis and constantly functioning in dozens of clinical trials.

Implementing Xavia-Clínicas required guaranteeing two computers at each of our clinical sites dedicated to compiling clinical trials data: obviously, you can't have the course of a trial interrupted because of a computer breakdown. So, although we have a spare, when one goes offline or presents problems, we move mountains to get replacement parts and ensure it is fixed as quickly as possible. On the connectivity front, Infomed improved connection speeds at our sites so the system would be easy and practical to use. The next piece of the puzzle is to get all patient clinical records (whether in clinical trials or not) digitized. To this end, MINSAP is launching a 


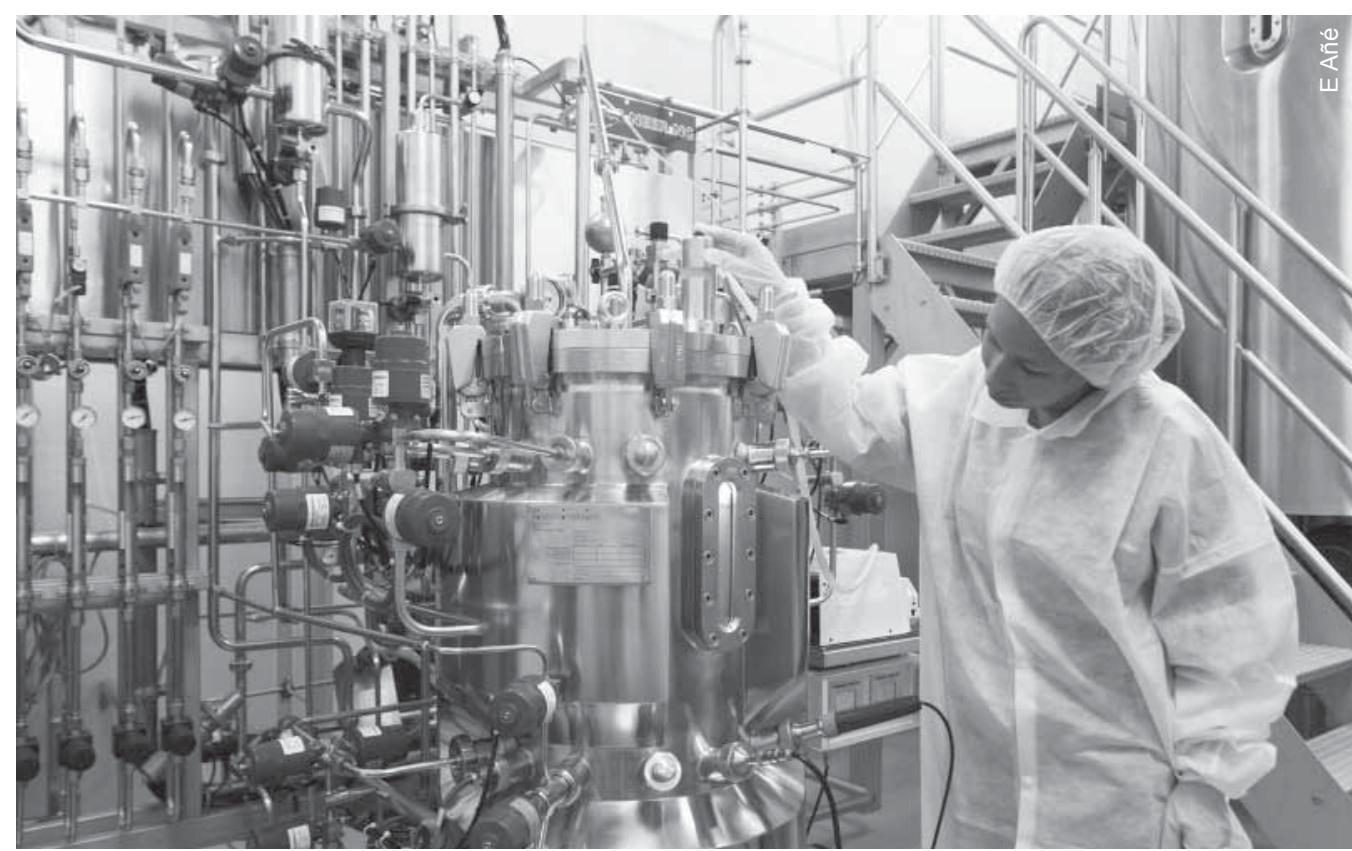

CIM production facilities, Havana

pilot project at Havana's Hermanos Ameijeiras Hospital, an intense process, but a huge step forward.

MEDICC Review: You are obviously passionate about your work. Can you talk a bit about where your energy and drive come from?

Tania Crombet: There's a contagious enthusiasm working here and this is thanks to our director Dr Agustín Lage. He's a real force and it's difficult not to be infected by his passion for the work. And the work itself is a huge motivator: we're researching cures and treatments for cancer. What scientist wouldn't want to be a part of this? Also, something that distinguishes Cuban research and perhaps is different from other contexts, is that we work together as a team. This isn't about individ- ual achievement or accolades. It's about improving health and quality of life.

MEDICC Review: One of CIM's flagship products, CIMAvaxEGF, a therapy for non-small cell lung cancer, is making news around the world, especially since the announcement of joint clinical trials proposed with Roswell Park Cancer Institute in the United States. Can you explain your role in its development and its use in Cuba?

Tania Crombet: First of all, the research, development, trials, and subsequent implementation of this therapy in the national health system were the result of a multidisciplinary team led by CIM clinical researcher Camilo Rodríguez. Obviously, I was a part of the team throughout the preclinical stage and monitoring of the trials, but the real handson work conducting the Phase III and IV trials, and introduction of CIMAvax-EGF into the health system, was carried out by the doctors and clinical trial specialists at our clinical sites.

What distinguishes CIMAvax-EGF from other CIM products that have been registered for use in Cuba is that it is administered in primary health care services - in community multispeciality polyclinics. This is possible because CIMAvax-EGF has been shown to be nontoxic, even with prolonged use, and doesn't interact negatively with other medications. It has proven safe and effective and improves quality of life for lung cancer patients.

Using this therapy in primary care requires close coordination among oncologists, immunologists, clinical trial specialists and

Molecular Immunology Center Human Resources, Research and Development Indicators

\begin{tabular}{|c|c|}
\hline Indicator & Number \\
\hline \multicolumn{2}{|l|}{ Human resources 2016* } \\
\hline Total number of workers & 1124 \\
\hline Average age of workers (years) & 38 \\
\hline Research scientists with higher degrees & 86 \\
\hline Women research scientists with higher degrees & 56 \\
\hline \multicolumn{2}{|l|}{ Research and development, December 1994-December 2015} \\
\hline Clinical trials conducted in Cuba & 160 \\
\hline Participants in domestic clinical trials & 32,000 \\
\hline Cubans who have received CIM treatments/medicines & 92,000 (1 in 120) \\
\hline CIM treatments/medicines registered in health system & 4 (nimotuzumab; CIMAvax-EGF; racotumomab; itolizumab) \\
\hline Registrations of CIM treatments/medicines abroad & 109 (more than one treatment in more than one country) \\
\hline Hospitals and polyclinics involved in CIM's clinical research & 147 \\
\hline Articles published in indexed journals & 200 \\
\hline Users trained on Xavia-Clínicas software platform & 600 \\
\hline Cuban provinces using Xavia-Clínicas for data collection and analysis & 15 (all) \\
\hline
\end{tabular}

Source: CIM, Departments of Clinical Research and Personnel, 2016

* Includes CIM branch in Santiago de Cuba 


\section{Interview}

others to assure Good Clinical Practices are being followed throughout the process. The dosage schedule for CIMAvax-EGF is a cycle of four doses every 14 days and then once a month thereafter; earlier, it was administered at oncology hospitals, but now patients can go to their local polyclinic, which is much easier for them. This year, MINSAP began designating one polyclinic in each municipality to administer CIMAvax-EGF. Our goal is to reach $100 \%$ coverage and lower nonsmall cell lung cancer mortality; to do this, we need to identify all patients requiring treatment. This is our next challenge.

\section{MEDICC Review: And the collabo- ration with the Roswell Park Can- cer Institute?}

Tania Crombet: This collaboration is an exciting opportunity, made possible by the efforts of Roswell Park to secure the required permission from the

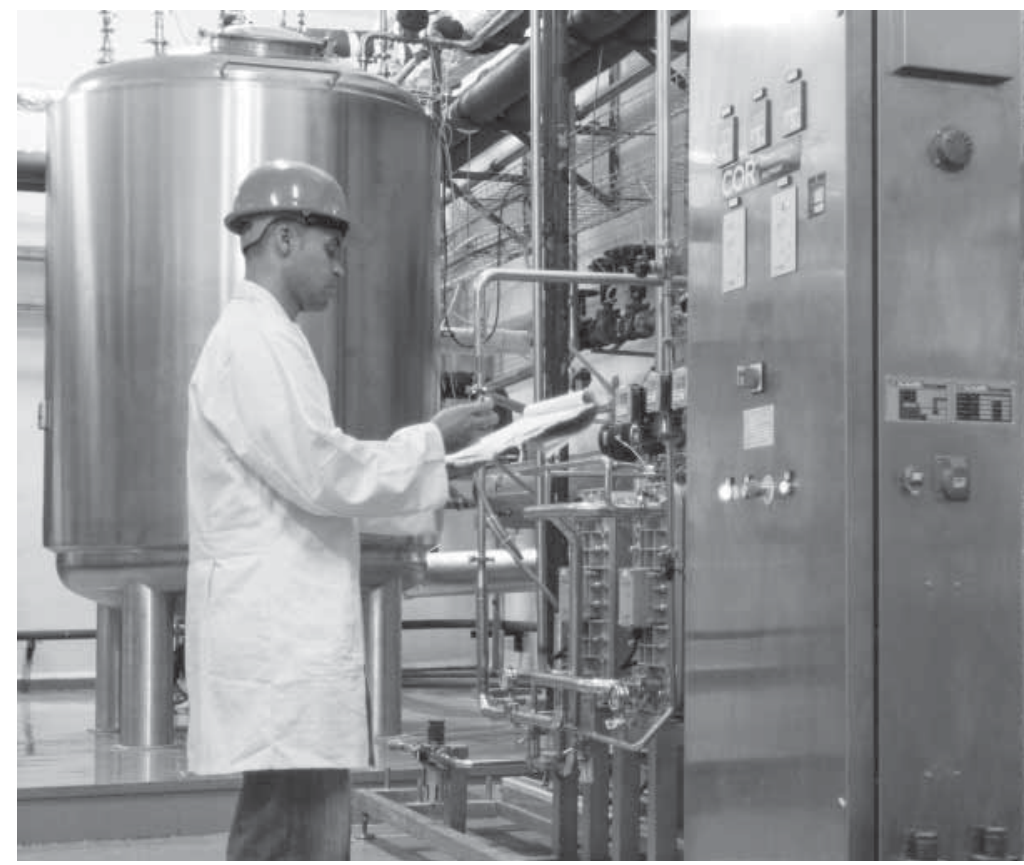

CIM production facilities, Havana
US Department of the Treasury's Office of Foreign Assets Control (OFAC) to import CIMAvax-EGF to the USA, the first step towards conducting joint clinical trials. CIM is providing CIMAvax-EGF, while Roswell Park is designing and implementing the study and assuming all clinical research costs. The next step is approval of the trial design by the FDA, which we are waiting for now. [On October 26, the The Buffalo News reported FDA approval for Phase I trials, as well as OFAC authorization for a joint venture company between the two institutions, the latter declared a "first" by Roswell Park CEO Candace Johnson.-Eds.]

MEDICC Review: Do you know how difficult a process it was for Roswell Park to secure permission to conduct clinical trials with CIM?

Tania Crombet: This isn't the first time a US institute has applied for and received OFAC permission to conduct clinical trials with a Cuban product. However, there is another hurdle specific to US-Cuba joint research: once a Cuban treatment successfully completes clinical trials in the United States and receives FDA approval, the US entity must apply for another, separate license from OFAC to be able to market and sell that product in the USA. And what if that second license is denied? Many companies don't want to risk investments in joint research or trials knowing they might be denied the license to sell it even when the product proves safe and effective. [On October 14, 2016, a Presidential order obliged OFAC to issue a general license for such activities, eliminating the specific and two-step licensing process.-Eds.] And, in many more ways, the US embargo is a real barrier to scientific collaboration between our countries. -1 -

Editors' note: For details on Cuba's clinical trial regulatory framework, public registry and results, see The ABCs of Clinical Trials in Cuba, MEDICC Review, July 2016, Vol 18, No 3, pp 9-14. www. medicc. org $/$ mediccreview/index. php? issue $=40 \& i d=546 \& a=v a$ 